\title{
Loss Differentiation and Congestion Control in Heterogeneous Networks
}

\author{
Junhua Huang ${ }^{\mathrm{a}}$, Zhaohua Long ${ }^{\mathrm{b}}$, Peng Zhou ${ }^{\mathrm{c}}$ \\ College of computer science and Technology, Chongqing University of Posts and \\ Telecommunications, Chongqing 400065, China;
}

a13618321784@163.com, blongzh@cqupt.edu.cn, czpjustu@163.com

\begin{abstract}
Keywords: Heterogeneous Networks, Packet Loss Classification, TFRC, Congestion Control, Kalman Filter.
\end{abstract}

\begin{abstract}
Aiming at the lack of effective packet loss differentiation mechanism in existing heterogeneous network congestion control strategies, which leads to efficient use of bandwidth, a packet loss differentiation mechanism based on Kalman filter is proposed and applied to TFRC congestion control strategy. In this algorithm, the average packet arrival delay in the stable phase and the packet arrival time interval in the packet loss phase are used to determine the type of the packet loss and the arrival interval is estimated by using Kalman filter to reduce the measurement error, and then the packet loss rate according to the packet loss type is recalculated. With the packet loss rate, the congestion control strategy starts to control the sender's rate. Experiments show that this algorithm can make congestion control policy more accurately identify the network status and improve the network utilization so that it can better meet the video streaming application requirements.
\end{abstract}

\section{Introduction}

With the development of wireless communication technology and multimedia technology, multimedia applications of mobile Internet are becoming more and more widespread [1]. Strict retransmission and acknowledgment mechanism in TCP congestion controls, and fluctuating transmission rate and delay jitter in AIMD congestion controls make them cannot meet the real-time video delay sensitive, high bandwidth, and packet loss tolerance, although data can be satisfied Transmission reliably. Therefore, some video transmission solutions use UDP protocol for video data transmission. However, due to the unreliable transmission of UDP, this will greatly reduce the quality of video transmission, making it unable to meet the demand [2]. The core of TCP, TFRC[3] and other transport protocols is congestion control, in wired networks, the lost packets will be measured as a sign of congestion in these mechanism, but in the wireless network, due to its high channel error code, losing packet because of the bit error in heterogeneous network video transmission cannot be ignored[4].

The traditional transport protocol lacks the effective packet loss differentiation mechanism and treats bit error packet loss as congestion packet loss, which result in lower transmission efficiency and higher transmission throughput.

\section{Related Work}

\subsection{Loss Differentiation}

At present, the loss differentiation is mainly based on end-to-end and link layer optimization. Because error packet loss can be well identified in the link layer, the cross-layer packet loss classification is better developed. However, it is more complicated to achieve the transformation in network nodes. End-to-end packet loss differentiation mainly included Biaz[5], SPLD[6], mBiaz[7], spike[8]. Biaz algorithm is achieved by calculate the packet arrival interval and the minimum packet arrival interval to distinguish the loss. But in the shared link, with the presence of multiple streams, the differentiation is low. SPLD uses the average accumulated packet arrival interval and the current packet arrival interval for packet loss differentiation, which performs well in single-stream and multistream, but the throughput is low. The spike algorithm differentiates packet loss by the one-way 
transmission delay and the current congestion state. But the spike algorithm cannot accurately distinguish between the congested packet loss and the random error packet loss.

\subsection{Kalman Filter}

Kalman filter [9] use the minimum mean square error as the best estimated criterion, to seek a recursive estimation algorithm. The basic idea is: using the signal and noise state space model, using the estimated value of the previous moment and the observed value of current moment to update the estimate of the state variable and calculate the estimate for the moment. The essence of Kalman filter is to reconstruct the state vector of the system from the measured values. It is recursive in the order of "Predicted-Measured-Modified", eliminating random interference based on system measurements and reproducing the state of the system.

\section{Algorithm Description}

In this paper, an improved congestion control strategy based on packet loss differentiation is proposed, which focuses on network error packet loss and congestion loss. At the receiving end, the Kalman filter is used to accurately estimate the packet-to-packet delay. By comparing the average accumulated packet interval delay with the current accumulated packet interval delay in steady state, the accuracy of the distinction and the performance of the congestion control protocol are improved.

The algorithm in this paper is improved based on SPLD, and use Kalman filter to improve the measurement accuracy of packet arrival interval delay, so as to better improve the discrimination accuracy and judge the network status more accurately.

The algorithm is shown in the figure below: The iat ${ }_{i}$ is the $\mathrm{i}$-th packet arrival time interval without packet loss in the current stage under the Kalman filtering, and iat stable $_{\text {means the cumulative arrival }}$ time interval of consecutive packets. The iat ${ }_{a v g}$ means the average packet arrival time interval, $\mathrm{n}$ is

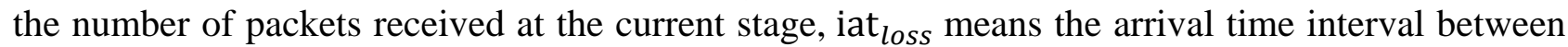
the previous packet and the latter one when packet losses, $\mathrm{m}$ means the number of packet loss detected when packet losses.

$$
\begin{gathered}
\text { iat }_{\text {stable }}=\sum_{i=\text { last }_{\text {atat }}}^{\text {now }} \text { iat }_{i} \\
\text { iat }_{\text {avg }}=\frac{\text { iat }_{\text {stable }}}{n-1} \\
\text { iat }_{\text {cur }}=\frac{\text { iat }_{\text {stable }+ \text { iat }_{\text {loss }}}}{n+m-2}
\end{gathered}
$$

The wireless error code package is sent from the sending end, and an error occurs due to channel reasons, which will cause a check error in the MAC layer of the receiving terminal and cannot make it be transmitted to the application layer. Therefore, wheniat ${ }_{\text {cur }} \geq$ iat $_{\text {avg }}$, the program considers that the cause of the packet loss event is due to wireless error code, otherwise it is congested to lose packets, and then clear the current state information and start a new phase.

The result of the packet loss differentation algorithm will eventually provide a more accurate classification of the network state for the congestion control policy, so as to satisfy the requirement of maximizing the throughput while satisfying the friendliness, as the congestion control strategy of the TCP may result in low throughput. Therefore, TFRC is used as congestion control protocol in this dissertation. It is quite fair to compete with the TCP stream for bandwidth, but throughput is much less variable over time than TCP, making it more suitable for streaming media applications characterized by relatively smooth delivery rates. 


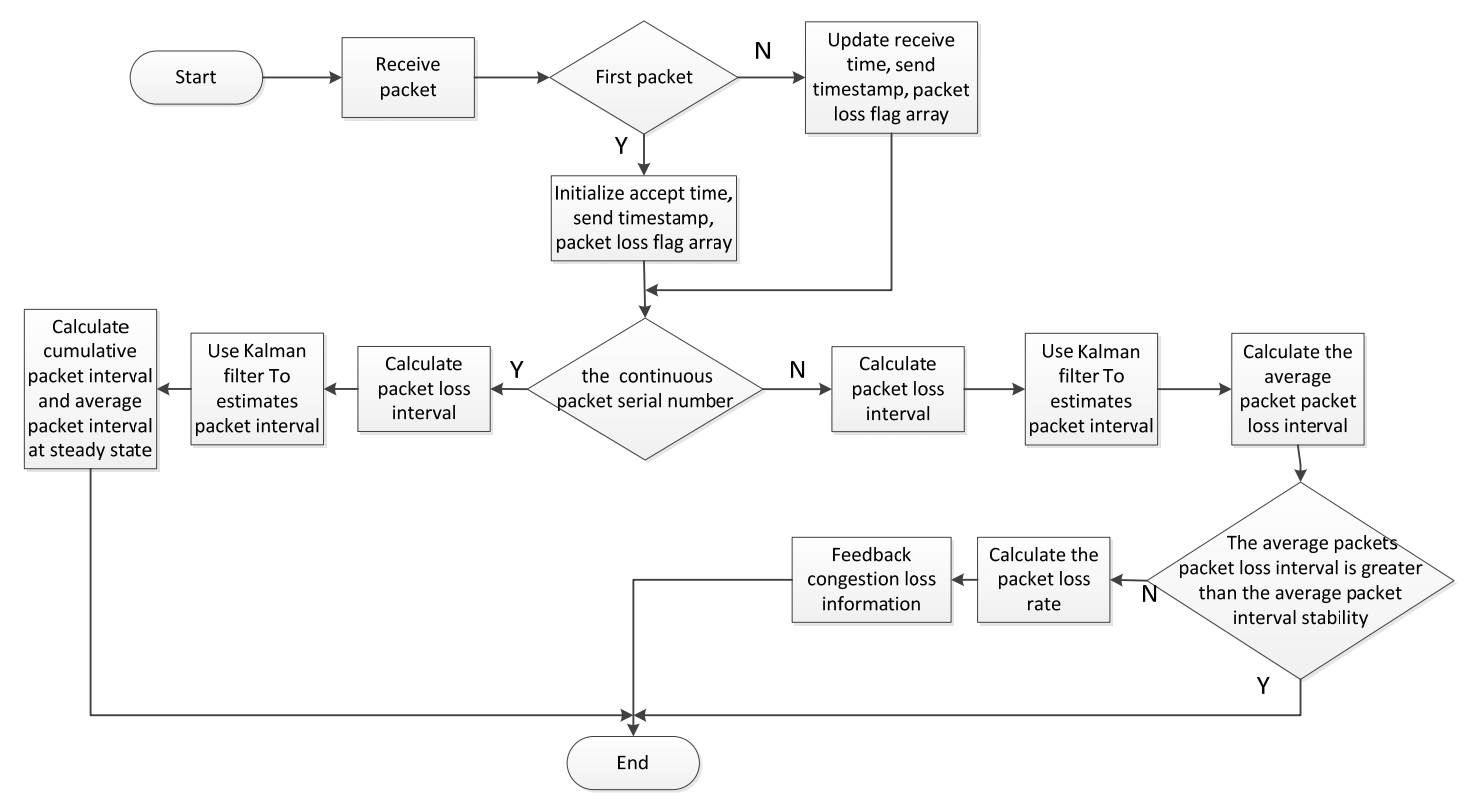

Fig. 1 the flowchart of loss differentiation algorithm

\section{Simulation}

In this paper, NS2 simulation software [10] is used to verify the effectiveness of the proposed algorithm. And then we make a comparsion with the existing mBiaz, Spld distinction algorithm, compared with no distinction between the TRFC algorithm algorithm. The performance of the algorithm has the following indicators to measure:

Throughput: Throughput is an important indicator to measure the effect of congestion control algorithm. The higher the throughput is, the better the congestion control effect is.

Misclassification rate: the Misclassification rate includes congestion loss as error packet loss mc and error packet loss as congestion loss mw, and the overall misclassification rate $\mathrm{mc}+\mathrm{mw}$.

Fairness: The purpose of the TFRC protocol design is to ensure the stable transmission of streaming media under the premise of being friendly with TCP. Therefore, the definition of fairness is the ratio between the two streams' throughput $\frac{\mathrm{Out}_{1}}{\mathrm{Out}_{2}} * 100 \%$

The experimental topology uses a wireless last top (WLH) network topology [4]. At the same time, the wireless link uses a random unified model to describe the network error behavior. Random unified model error the data in the form of random scores, the average loss rate $\mathrm{p}$ on behalf of the packet loss rate.In order to facilitate the calculation, this paper uses the first-order Kalman filter, that is, where A is a constant $1, \mathrm{H}$ is also a constant 1.

It can be seen from the figure 2 that compared with mBiaz and SPLD, the loss differentiation algorithm KSPLD proposed in this paper has greatly improved in the misclassification rate. The misclassification rate of the SPLD remains at around 0.6, while the KSPLD algorithm has the average error with a resolution of only 0.3. The efficiency is nearly doubled in the SPLD differentiating algorithm and the original TFRC algorithm. The accurate packet loss distinguishing makes the TFRC maximize the network efficiency, accurately identify the error code and prevent the misjudgment leading to the decrease of the throughput too fast in our purposed algorithm. The proposed algorithm can maintain high throughput under single flow conditions from the figure 2.a.

From the figure 3, we can know that, due to the existence of multiple streams, the misclassification rate of each algorithm appears a certain amount of jitter. However, compared with the SPLD classification algorithm, the KSPLD classification algorithm proposed in this paper can maintain a misclassification rate. While the throughput can also be reflected from the figure that in the lower misclassification rate result to the higher throughput. 


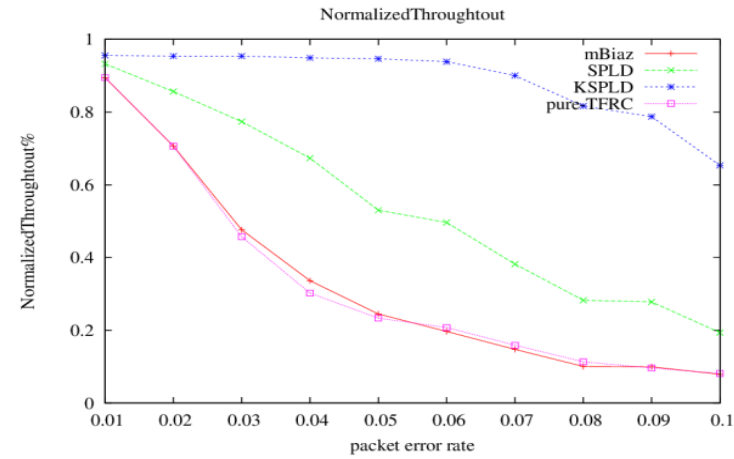

(a)normal throughout

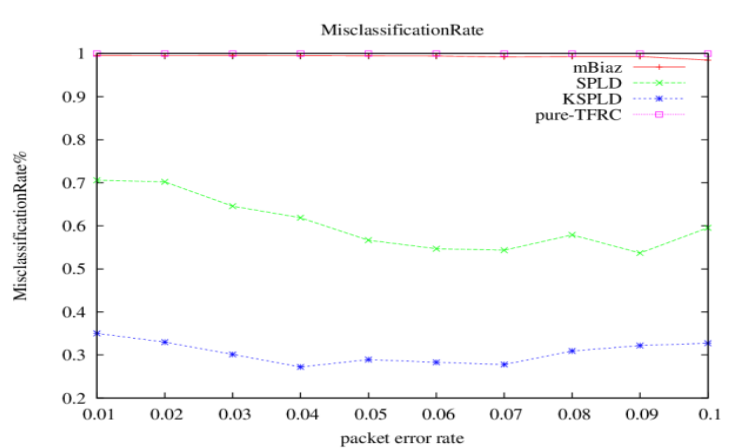

(b) misclassification rate

Fig.2 Simulation results for single flow

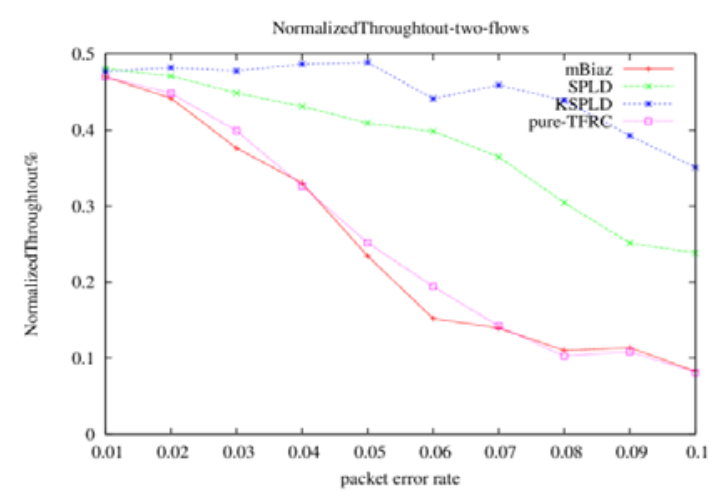

(a)normal throughout

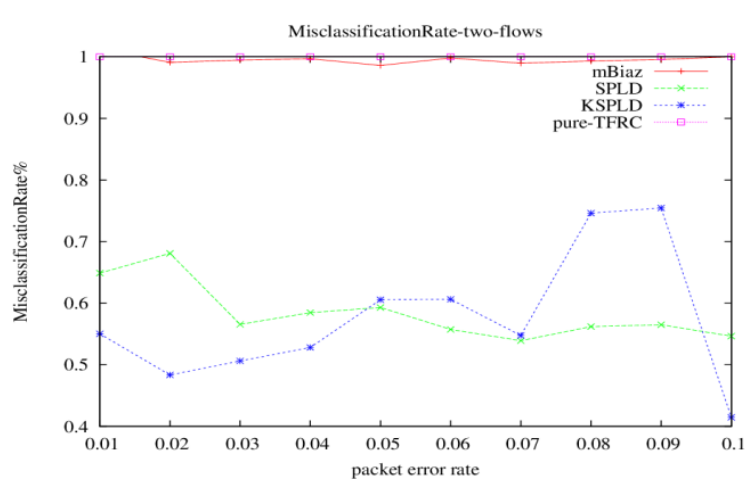

(b) misclassification rate

Fig.3 Simulation results for double flows

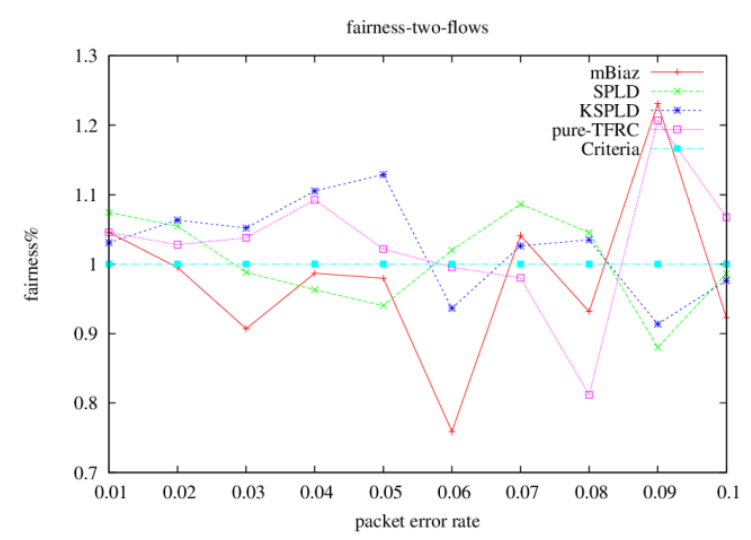

Fig.4 fairness for two flows

In order to judge the fairness, this article uses the flow of the same differentiation algorithm to compare the throughput. According to the figure 4, in the case of high throughput, the algorithm proposed in this paper can also meet the requirements of fairness, making the multi-flow more fully utilize the network bandwidth.

\section{Summary}

In this paper, we propose a packet loss classification algorithm based on Kalman filter. The algorithm estimates the average packet arrival delay in the stable phase and the packet arrival time interval in the packet loss phase to classify the packet loss type, and recalculate the packet loss rate.

Based on this differentiating algorithm, we combine the TFRC protocol and make the simulation experiment based on it. According to the experimental results, we can conclude that the proposed congestion control improvement strategy can better adapt to heterogeneous networks and more 
accurately distinguish between different packet loss type, so that the sender has a more accurate perception of the network and improve network throughput.

\section{References}

[1]. Yubao Jin, Yuning Dong. Packet Loss Differentiation and Congestion Control Mechanism in Heterogeneous Networks. Journal of Applied Sciences. Vol.33 (2015) No. 03, p.234-242.

[2]. Gaetano Carlucci, Luca De Cicco, Cesar Ilharco,et al. Congestion Control for Real-time Communications: a comparison between NADA and GCC. Mediterranean Conference on Control and Automation. Athens, Greece, June 2016 p.575-580.

[3]. S. Floyd, M. Handley, J. Padhye, et al. Equation-based congestion control for unicast applications. Proc. ACM SIGCOMM. Stockholm, Sweden, Aug. 2000, p.43-56.

[4]. Hyungho Lee, Chong-Ho Choi. A Loss Discrimination Scheme for TFRC in Last Hop Wireless Networks. Wireless Communications and Networking Conference. Kowloon, China. March 2007, p, 3082-3086.

[5]. S. Biaz, N. H. Vaidya. Discriminating Congestion Losses from Wireless Losses using InterArrival Times at theReceiver. Application-Specific Systems and Software Engineering and Technology. Richardson, TX, USA, March 1999 p.10 - 17.

[6]. Min Kyu Park, K. H. Sihn, Jun Ho Jeong. A Statistical Method of Packet Loss Type Discrimination in Wired-Wireless Networks. Consumer Communications and Networking Conference. Las Vegas, NV, USA, Jan. 2006, p. 458 - 462.

[7]. Song Cen, P. C. Cosman, G. M. Voelker. End-to-End Differentiation of Congestion and Wireless Losses. IEEE/ACM Transactions on Networking. Vol.11 (2003) No. 5, p. 703 - 717.

[8]. Y. Tobe, Y. Tamura, A. Molano, S. Ghosh, et al, Achieving moderate fairness for UDP flows by path-status classification. IEEE Conf. Local Computer Networks. Tampa, FL, Nov. 2000, p. 252261.

[9]. Information on: http:/ /www. cs.unc.edu/ welch.

[10]. Zhiheng Ke, Rongxiang Cheng, Dejun Deng. NS2 simulation experiments one multimedia and wireless network communications. Electronics Industry Press, 2009, p.235-243. 